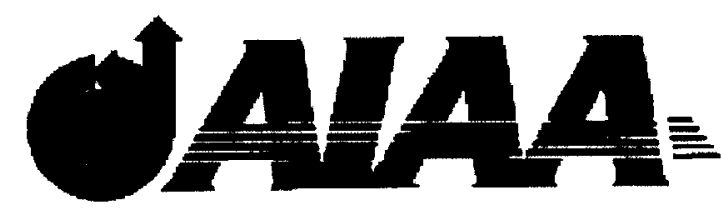

\title{
AIAA 2002-0440 \\ IMPROVEMENT OF FLOW QUALITY IN \\ NAL CHOFU MACH 10 NOZZLE
}

John Lacey

Aero Systems Engineering, St. Paul, Minn, USA

Dr. Yasutoshi Inoue

NAL Chofu, Tokyo, Japan

Akio Higashida

Mitsubishi Heavy Industries, Kobe, Japan

Manabu Inoue

Mitsubishi Heavy Industries, Tokyo, Japan

Dr. Kouichi Ishizaka

Mitsubishi Heavy Industries, Takasago, Japan

Dr. John J. Korte

NASA Langley Research Center, Hampton, VA USA

\section{0th AIAA Aerospace Sciences Meeting \& Exhibit 14-17 January 2002 / Reno, NV}

For pormleaton to copy or republiah, contect the copyrtight owner named on tho firat pege. For AlAA-hold copy-

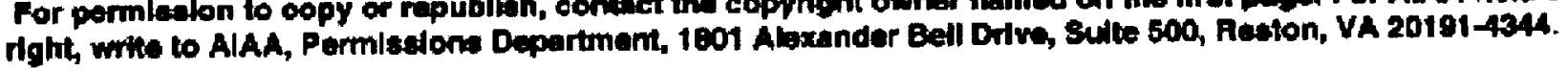




\title{
IMPROVEMENT OF FLOW QUALITY IN NAL CHOFU MACH 10 NOZZLE
}

\author{
John Lacey* \\ Aero Systems Engineering, St. Paul, Minn, USA \\ Dr. Yasutoshi Inoue** \\ NAL Chofu, Tokyo, Japan \\ Akio Higashida (Kobe), Manabu Inoue (Tokyo) and Dr. Kouichi Ishizaka (Takasago) \\ Mitsubishi Heavy Industries, Japan \\ Dr. John J. Korte**
NASA Langley Research Center, Hampton, VA USA
}

\section{SUMMARY}

As a result of CFD analysis and remachining of the nozzle, the flow quality of the Mach 10 Hypersonic Wind Tunnel at NAL Chofu, Japan was improved. The subsequent test results validated the CFD analytical predictions by NASA and MHII.

\section{INTRODUCTION}

The 1.27 meter diameter Mach 10 nozzle at the National Aerospace Laboratory (NAL) at Chofu, Japan was designed in the early 1990 s for a nominal operating condition of $1070 \mathrm{~K}$ and $6 \mathrm{MPa}$. This nozzle was designed and manufactured in accordance with industry standards at the time. The flow quality (see Figure 1) of this project was acceptable at the time '; but since then, the standards of the industry have been tightened ${ }^{2}$, and the Chofu design was not up to recent world-class performance criteria ${ }^{3}$.

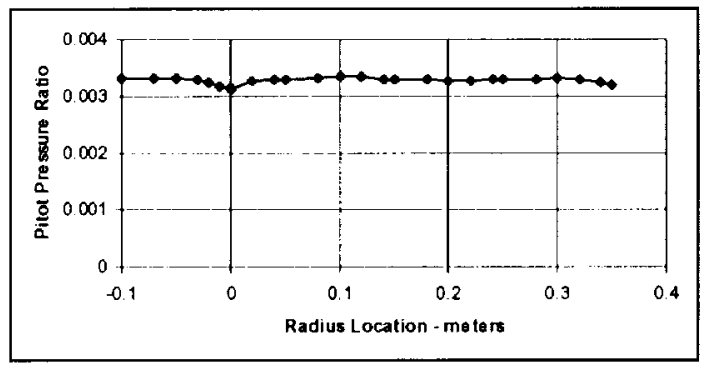

Figure 1 Pitot Pressure Profile - Center of the Test Section
A program was initiated to improve the flow quality through the following steps:

1) CFD modelling of the existing flow,

2) Development of possible nozzle contours,

3) Evaluation of these nozzle contours

4) Remachining of the nozzle, and

5) Validation testing.

Mitsubishi Heavy Industries (MHI), Aero Systems Engineering (ASE), and NASA Langley participated in this effort with NAL. MHI did the contour measurements and remachining; MHI and NASA LaRC used their CFD capability to model the original test results and evaluate the new contours; and ASE collaborated to develop the improved nozzle contours.

\section{ANALYSIS - EXISTING CONTOUR}

The initial test data shown in Figure 1 was expanded to include data at several other axial stations in the test section. As shown in Figure 2, the data indicate pressure waves traversing the test section and the effects of other waves converging on centerline. The spread of Pitot pressure ratio is $\pm 7 \%$; this implies a Mach Number variation of $\pm 1 . \overline{4} \%$. This profile was shown to be nearly symmetrical.

Through a preliminary CFD analysis these waves were speculated to originate from the nozzle wall as shown in Figure 3

** Senior Member AIAA * Member AIAA

Copyright $\triangle 2002$ the Annerican Institute of

Aeronautics and Astrontutics Inc. All rights reserved 


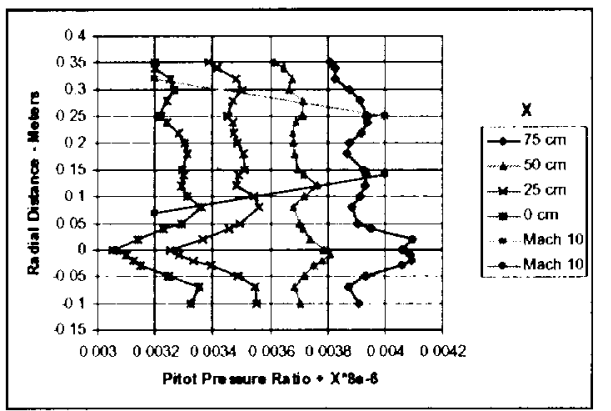

Figure 2 Pitot Pressure Profiles - Various axial stations

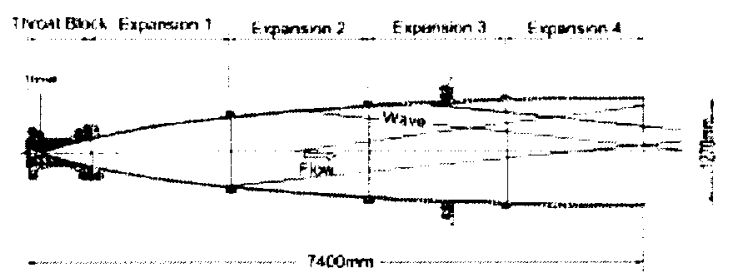

Figure 3 Nozzle Sections and Possible Wave Pattern

While one wave could come from the joint between Expansion 1 and Expansion 2, the others were not so obvious. Those others could be reflections of waves that originated further upstream or they could be waves generated on the nozzle wall at the location shown. Therefore, the wall contour was precisely measured at many stations so small disturbances could be detected. The results of these measurements showed that the contour was, in general, a shape typical of hypersonic nozzles but the slope and curvature were not fully smooth as shown in Figure 4 for the throat and initial expansion.

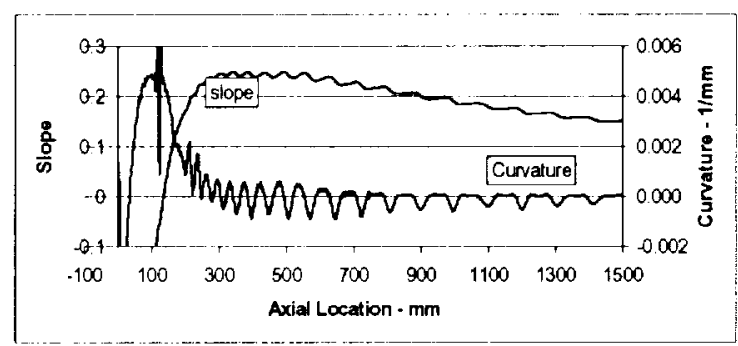

Figure 4 Original slope and curvature

The complete measured contour data was placed in both the NASA-Langley ${ }^{4,5,6}$ and MHI ${ }^{7,8,9}$ CFD models and the predictions are shown in Figure 5 and Figure 6 respectively. In each case, the general character of the non-uniform profiles is predicted.
(The differences that do exist are not deemed to be significant because a prediction of such steep gradients is subject to exact measurements of all peaks and valleys and exact duplication of flow properties over the entire flow field. While the coordinates of the contour were measured in fine detail, there is the possibility that some peaks/valleys were not recorded and/or that some gas properties are not fully modeled.)

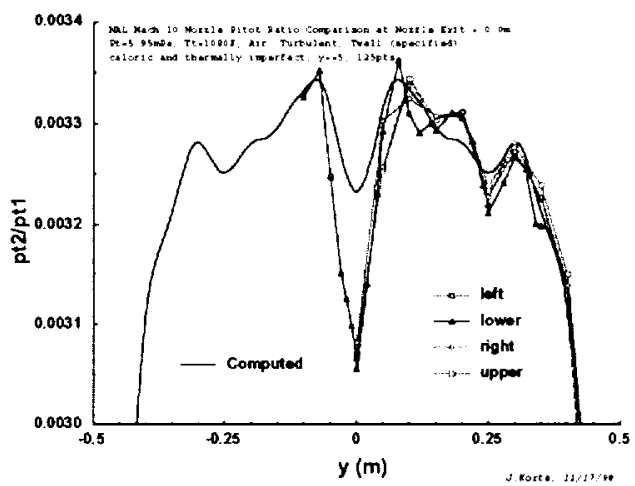

Figure 5 Comparisons of Pitot Pressure Profiles with CFD - NASA Langley

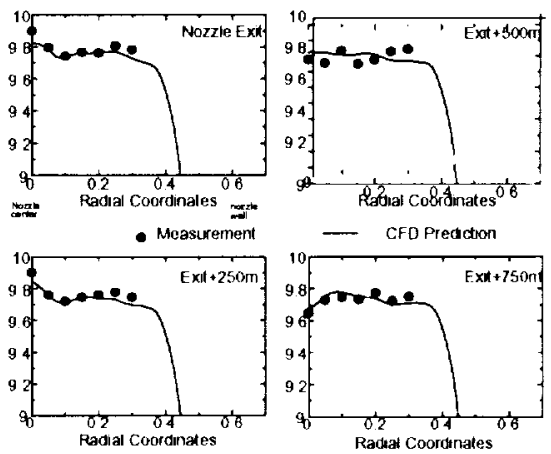

\section{Figure 6 Comparisons of Mach Number Profiles with CFD - MHI}

\section{OTHER TOOLS FOR ANALYSIS}

Along with the Pitot pressure profiles, the usual contour maps of Mach, pressure, velocity, etc. were also obtained (see Figure 7 for an example). The origins of waves were not easily interpreted from these usual contour plots and the parameter "GradP/P" was developed. These contours are shown in for the original nozzle. As shown in Figure 8, many waves that come from the wall can be seen. Some of these waves get cancelled through various interactions and a few waves enter the test section. Two of these waves generally 
agree with the two pressure waves that were identified in Figure 2. The strong gradients on the centerline are also obvious in this Figure 8 . Contours could be analyzed more easily on the basis of this CFD parameter.

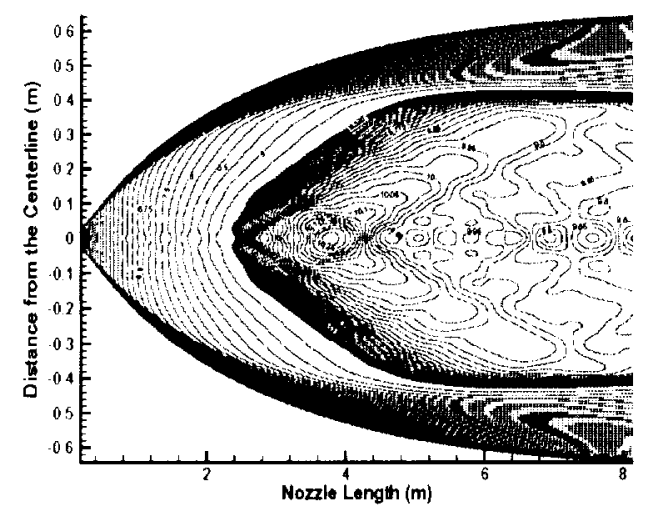

Figure 7 Typical Mach Contour

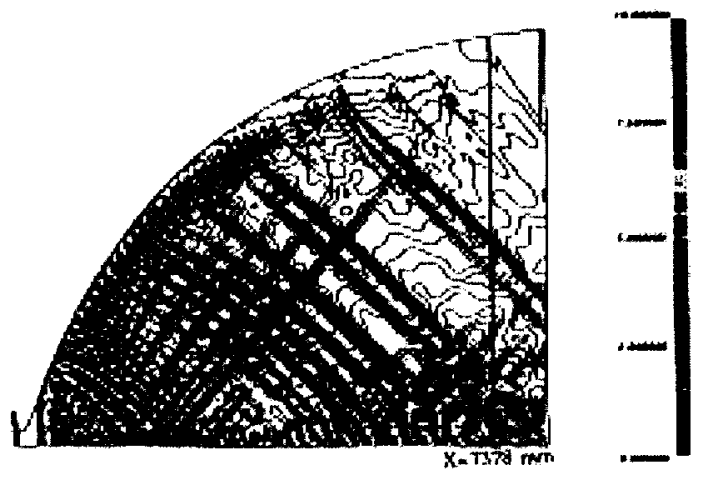

Figure 8 Contour of GradP/P for Original Nozzle

\section{CONTOUR SMOO'TIING}

\section{Initial Smoothing}

Based on the successful validation of the CFD models, new contours were developed in an attempt to obtain more uniform flow. One of the criteria for these new contours was that most of the existing nozzle had to be re-used in order to minimize cost. The sections of the nozzle are shown in Figure 3.

The first attempt was to smooth just the throat block; that is, the initial expansion of the nozzle. This is a small section and could be made new for relatively low cost. The results are shown in Figure 9. Note that in both the cases that were studied, major non-uniformities still remain. In this case, it does not seem possible to achieve uniform flow with modification of only the throat block.

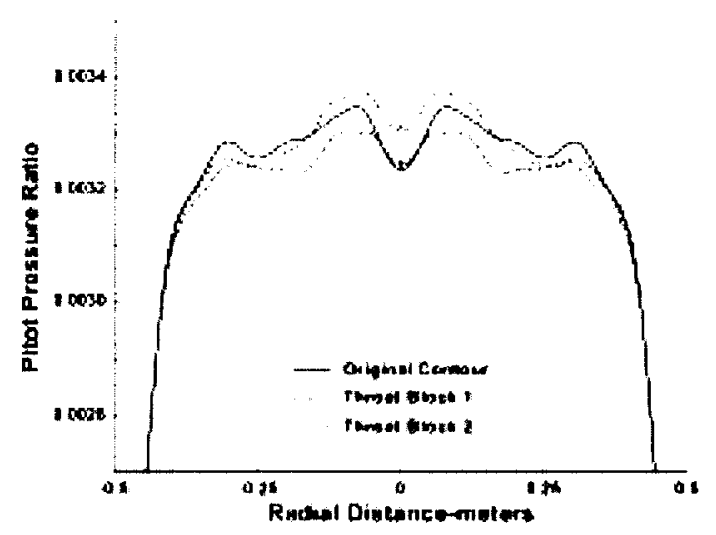

Figure 9 Pitot Pressure Profile for Two Cases of Throat Block Smoothing - Compared with Original

\section{Final Smoothing}

Further attempts to obtain uniform flow consider the entire nozzle, not just the throat block. The original contour was based on the Method of Characteristics plus Boundary Layer correction (MOC+BL) and was then smoothed using cubic spline methods. For the new coordinates, a revised smoothing method was used based on polynomial equations of nozzle radius as a function of nozzle axial location. The nozzle was divided into axial sections for the curve fit procedure In each section a $5^{\text {th }}$ order polynomial equation was applied. The polynomials were of the $5^{\text {th }}$ order since this allowed the necessary inflections of the curvature. (For reference, a cubic equation has a linear second derivative, i.e. curvature, and use of several cubic equations would have a discontinuous third derivative, not the smooth charateristic needed for a nozzle.) The lengths of the sections were adjusted to provide a good fit of the existing coordinates. Five sections were needed as shown in Figure 10.

At the match point between each section, the coefficients were adjusted until there was a match for nozzle Radius, Slope, and Curvature. The section from about $2500 \mathrm{~mm}$ to $5500 \mathrm{~mm}$ is covered by a single equation. This is a subtle but important point; any wave -originating in this range goes directly to the core of the test section, virtually without attenuation. This section must be a very smooth and this is best provided by a single equation.

4 


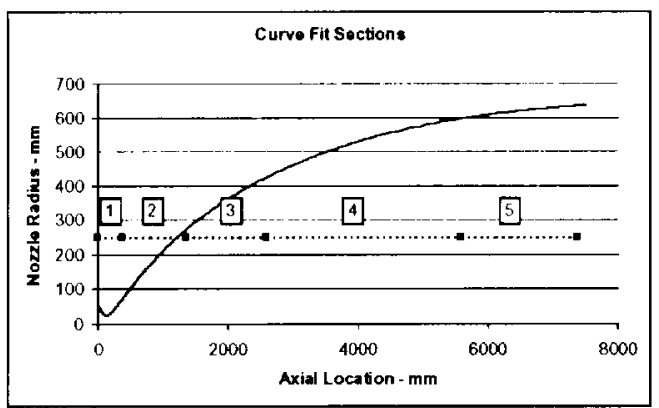

Figure 10 Sections of Nozale for Curve Fit Procedure

Further, since the MOC+BL method is step-by-step process that uses assumptions and averages to compute the downstream points, it is possible that the best coordinates might be slightly different than the computed values. Therefore, exact matching of the MOC $+B L$ coordinates was not a priority in this smoothing process. It is more important to have a continuous third derivative (smooth curvature) than to exactly match the MOC $+3 \mathrm{~L}$ computed points.

One criteria for this nozzle was that the existing surface had to be machined at least $0.5 \mathrm{~mm}$ so that the new contour could be made with confidence. This allowance was needed for the tolerances of machine setup, concentricity, and a full removal of the existing coating. This was accomplished by rotating the new contour about a point upstream of the throat. (The rotation was only 0.0005 radians; additional smoothing was done in the throat area to maintain the throat size.)

\section{NEW CONTOUR}

The above method was used to generate several nozzle contours that might improve the flow uniformity. Each contour was evaluated using the CFD already validated to the test data as mentioned above.

The final selected change in contour is shown in Figure 11. This shows the slight modification that was applied to the original nozzle. Note that, exclusive of the general downward trend (resulting from the rotation mentioned above), the contour change is only about $\pm 0.25 \mathrm{~mm}$. The peaks in the line at about 3400 and $5000 \mathrm{~mm}$ indicate compression corners (as compared to the new contour) and they nearly match the origin of waves suggested in Figure 3 . At each of these two locations, the slope is changed by about 1.6 $\mathrm{mm} / 1000 \mathrm{~mm}(\sim 0.1 \mathrm{deg}$ total change); this highlights the need to limit the size of slope variations, especially in the compression mode. Note that the sharp compression corner near $700 \mathrm{~mm}$ may have contributed to the problem on centerline (after several reflections); for reference see Figure 8 that shows several strong waves originating in this area.

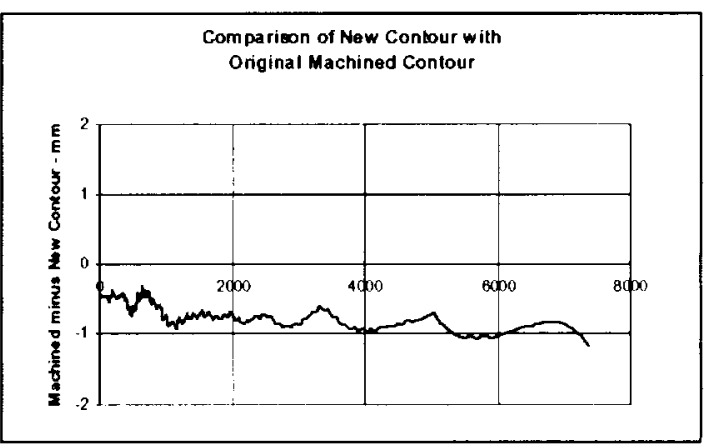

Figure 11 Change in Contour

\section{MACHINING RESULTS}

The results of the machining to the new contour are shown in Figure 12. The contour was held to within nearly,$+-0.025 \mathrm{~mm}$ of the design values. The resulting compression corners near the joints at 2500 and 5800 $\mathrm{mm}$ are less than $0.01 \mathrm{deg}$. There is a step, about 0.03 $\mathrm{mm}$ high, near 2500 and a similar gap near $4100 \mathrm{~mm}$. As will be shown later, the flow quality is quite good despite these deviations.

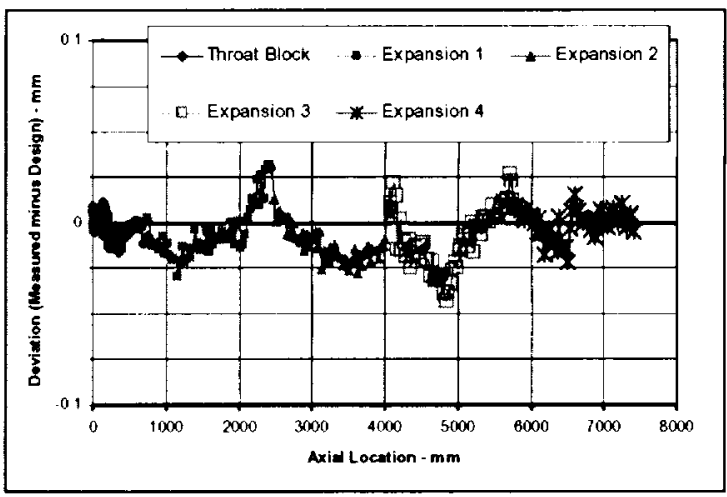

Figure 12 Results of Machining to New Contour

\section{TEST RESULTS}

Pitot Pressure

Several improvements were made in the measurement apparatus prior to conducting the final tests. The number of Pitot probes was increased and the span extended so that measurements are at $10 \mathrm{~mm}$ transverse 
spacing and the measurements now extend into the boundary layer. The test data was taken at more axial stations, with $50 \mathrm{~mm}$ increments in the axial direction covering $750 \mathrm{~mm}$, in both the horizontal and vertical planes including tests with off-axis placements (again both in the vertical and horizontal orientations). The data is summarized in Figure 13; note that the spread of Pitot pressure within the core $\pm 350 \mathrm{~mm}$ around centerline has been reduced from $\pm 7.5 \%$ to $\pm 1.5 \%$. This implies that the Mach Number variation is now been reduced from $\pm 1.5 \%$ to $\pm 0.3 \%$.

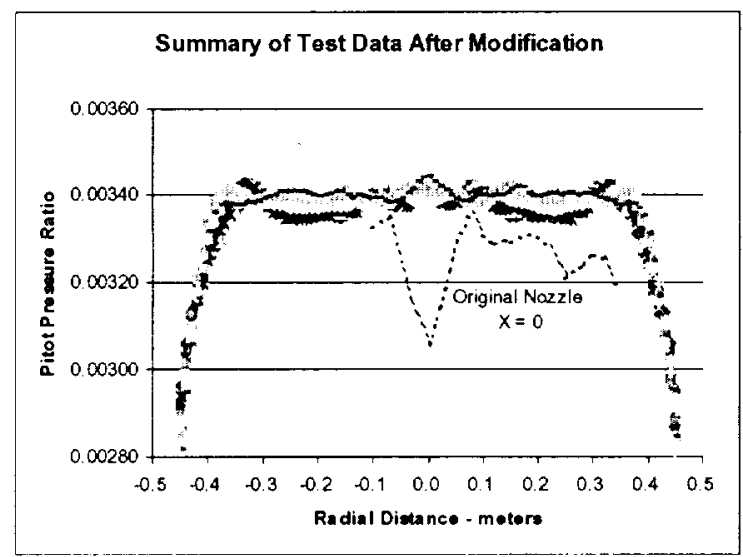

Figure 13 Final Test Results

\section{Flow Angle}

The flow angularity was measured using a rake with conical probes. The measurements cover $0.72 \mathrm{~m}$ in width with 7 conical probes spaced $0.12 \mathrm{~m}$. These tests were made in 10 axial locations, every $0.1 \mathrm{~m}$ from nozzle exit $(X=0)$ to $0.9 \mathrm{~m}$ downstream, with the rake in both the vertical and horizontal position, totaling 130 points. With the rake in a horizontal position, the distribution of flow angle was obtained in 7 vertical locations $(Z=0, \pm 0.12, \pm 0.24, \pm 0.3 \mathrm{~m})$ at 3 longitudinal locations $(X=0,0.25$, and $0.5 \mathrm{~m}$ ), totaling 147 points (some duplicating the longitudinal positions).

The flow inclination angles were from -0.02 degrees to 0.1 degrees on the centerline and within \pm 0.2 degrees in the whole core flow region. An example of the result at the nozzle exit cross section is shown in Figure 14. The flow is nominally axisymmetric with slight inward flow at this location.

\section{Centerline Mach Number}

Further evidence of the flow quality achieved in this effort is shown in Figure 15 (taken from Reference 10). This shows the comparison of the centerline Mach number in this wind tunnel as compared to the same parameter in the AEDC Tunnel C. The long length of nearly constant Mach number in the NAI, tunnel is apparent.

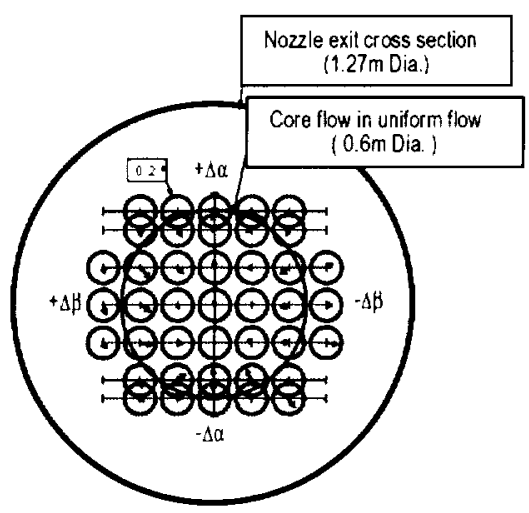

Figure 14 Flow inclination angle distribution at nozzle exit cross section

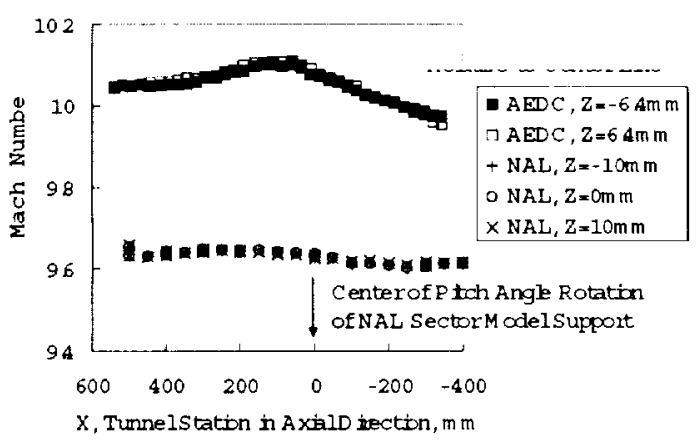

Figure 15 Centerline Mach Number Variation Compared with AEDC Tunnel C (from Reference 10).

\section{CONCLUSION}

An international team has studied the flow quality of the NAL Mach 10 nozzle. The participants used both experimental data and computational methods to identify concerns and to modify the nozzle contour. The results with the new contour show that the flow quality has been significantly improved. As a result the NAL Mach 10 wind tunnel can now serve the aerodynamic community as a world-class testing facility.

\section{ACKNOWLEDGEMENT}

The authors wish to acknowledge the contributions of the following people that also contributed to the project and this paper; namely Mr. Seizo Sakakibara and Dr. Koichi Hozumi of NAL Chofu. 


\section{REFERENCES}

1) Boudreau, A.H., "Performance and operational characteristics of AEDC/VKF tunnels $A, B$, and C," AEIDC-TR-80-48 (1981).

2) Micol, J. R., "Hypersonic Aerodynamic/Aerothermodynamic Testing Capabilities at Langley Research Center: Acrothermodynamic Facilities Complex," AIAA-95-2107.

3) Aerodynamics Department, "Plan and Structure of Large-size Hypersonic Wind Tunnel," Technical Report of NAL, TR-1261 (1995). (in Japanese)

4) Korte, J. J., Kumar, A., Singh, D. J. and White, J. A., "CAN-DO - CFD-Based Alerodynamic Nozzle Design \& Optimization Program for Supersonic/Hypersonic Wind Tunnels," AIAA Paper 92-4009, July 1992.

5) Korte, J. J., Hedlund, E. and Anandakrishnan, S., "A Comparison of Experimental Data With CFD For The NSWC Hypervelocity Wind 'Tunnel $\$ 9$ Mach 14 Nozzle," AIAA Paper 924010, July 1992.

6) Korte, J. J. and Hodge, J. S., "Flow Quality of Hypersonic Wind-Tunnel Nozzles Designed Using Computational Fluid Dynamics," $J$. of Spacecraft and Rockets, Vol. 32, No. 4, JulyAugust 1995, pp. 569-580

7) Ishizaka, K., Ikohagi, T. and Daiguji, H., "A High Resolution Finite-Difference Scheme for Compressible Gas-Liquid Two Phase Flows," Proc. of the 5th ISCFD, Vol.1(1993), pp.352357

8) Figgers, Jr. A. J., "One-Dimensional llows of an Imperfect Diatomic Gas,” NACA Report 959, 1950

9) AMES Research Staff, "Equations, Tables And Charts For Compressible Flow," NACA TR 1135,1953

10) Nagai, S., Tsuda, S., Koyama, T., Hirabayashi, N., and Sekine, II., "Comparison of Winged Vehicle Force Data at Large Hypersonic Wind Tunnels", AIAA-2001-0166. 